\title{
Proposta de Desenvolvimento de Sistema de Automação de Irrigação por Microaspersão utilizando Hardware Livre
}

\author{
Mauro J. Pereira, José S. Filho, Jonatas M. G. Cruz, Jime R. Ribeiro, André A. P. \\ Carvalho \\ Laboratório de Computação Aplicada - LCA \\ Instituto Federal do Pará (IFPA) - Campus de Cametá \\ 68400-000 - Cametá - PA- Brasil \\ madjepe@gmail.com, jose.sousa.filho@gmail.com, jhon.mgc@gmail.com, \\ engenheirajime@gmail.com, andrepcarvalho@gmail.com
}

\begin{abstract}
This paper describes a proposal for the development of an irrigation system focused on the cultivation of açai palm (Euterpe oleracea Mart.) based in micro sprinkler for small and medium farmers, especially in the Baixo Tocantins region. The results of this research cover, in this first moment, a survey and pre-design of the main components and modules of the proposed system.
\end{abstract}

Resumo. Este artigo descreve uma proposta de desenvolvimento de sistema de irrigação do cultivo de palmeiras de açaí (Euterpe oleracea Mart.) por microaspersão voltado para pequenos e médios agricultores, especialmente da região do Baixo Tocantins. Os resultados desta pesquisa abrangem, neste primeiro momento, um levantamento e pré-projeto dos principais componentes e módulos do sistema proposto.

\section{Introdução}

A região do Baixo Tocantins, pontualmente na microrregião do município de Cametá, abrange cinco municípios, dentre eles: Cametá, Mocajuba, Baião, Limoeiro do Ajuru e Oeiras do Pará, conforme Figura 1. Esta região é formada basicamente por micro e pequenos proprietários rurais, cuja estrutura produtiva está alicerçada em atividades de subsistência e também no agroextrativismo.

Dentre as principais atividades extrativistas realizadas, destaca-se a coleta do açaí (Euterpe oleracea Mart.), que é a mais praticada (HOMMA, 1989, 1993). Esta cultura é encontrada em abundância, principalmente nas áreas de várzea, o que acaba caracterizando o perfil produtivo desta região.

Contudo, mesmo com um enorme potencial para obtenção da polpa e suco do açaí, principais produtos oriundos desta atividade, a quantidade produzida ainda não é suficientemente capaz de abastecer os principais comércios regionais, com destaque para o município de Belém (capital do Estado do Pará) que constitui o principal mercado consumidor (GUIMARÃES, SANTOS, RODRIGUES, \& FRAHA, 2004), tão pouco os de escala nacional e internacional.

Aliado a esta problemática, em décadas passadas, a exploração desordenada de áreas com açaizeiros nativos (várzea), principalmente para retirada do palmito, levou a uma redução da oferta e aumento do preço do fruto. Logo, faz-se necessário expandir e 
avançar esta cultura para outras áreas produtivas com vistas a suprir a demanda real de consumo de produtos oriundo do açaí.

Neste contexto, por se tratar de uma cultura originalmente encontrada em regiões de várzea, como alternativa eficiente, pergunta-se: "como esta cultura poderia ser implantada em terra firme?". Tal afirmação sustenta-se em várias experiências exitosas realizadas pela EMBRAPA com o cultivo do açaí em terra firme, na qual, inclusive, originou variedades de produções adaptadas a este ecossistema diferenciado .

Considerando o acima, pode-se afirmar que a busca por modelos sustentáveis capazes de alinhar alternativas produtivas com a conservação ambiental é um desafio árduo. Entretanto, com boas práticas de manejo dos ecossistemas, torna-se possível obterse produtos de qualidade e ao mesmo tempo adotar práticas que minimizem os impactos no meio ambiente.

Deve-se considerar a tecnologia como uma ferramenta importante que contribui para o sucesso de modelos sustentáveis. Porém, encontrar mecanismos eficientes que utilizem meios naturais para monitorar e aprimorar processos produtivos ainda é raro, principalmente porque projetos com este lastro possuem alto custo de implantação e manutenção (ROSITO, 2014) (COSTA, 2013). Motivo pelo qual, a proposta aqui descrita constitui programar um protótipo de automação em áreas de plantio de açaí.

A referida proposta será uma miniestação equipada com sensores (umidade do solo, umidade relativa do ar e temperatura do ar) que orientará as tomadas de decisões do equipamento quanto ao momento adequado em que o sistema de irrigação irá ligar ou desligar, reduzindo assim custos com energia elétrica.

Deste modo, o sistema computacional aliado com o hardware adequado automatizará o sistema de irrigação por microaspersão, partindo-se de variáveis ambientais que monitorariam e gerenciariam todo o processo. Este protótipo também será um data logger, que além de automatizar o sistema, gerará e armazenará informações importantes que servirão de base, inclusive, para propor uma metodologia de irrigação adequada a cultura do açaí adaptada ao microclima da região do Baixo Tocantins.

\section{Trabalhos Correlatos}

Nesta seção apresentara um comparativo entre alguns trabalhos atuais sobre a criação de estações meteorológicas e suas aplicabilidades, além de apresentar de que maneira tais trabalhos se diferenciam da proposta aqui defendida.

Costa (2013) apresenta o desenvolvimento de uma estação meteorológica, para monitoramento de temperatura, pressão atmosférica, umidade relativa do ar, velocidade e posição do vento. Os sensores utilizados fornecem dados meteorológicos em determinado intervalo de tempo para um sistema microcontrolado que processa tais informações e as envia para um computador, através de uma interface de comunicação serial baseada no protocolo $R S-232$, permitindo que o usuário armazene e visualize estas informações em um terminal de comunicação.

Rosito (2014), por sua vez, apresenta um projeto que propõe o estudo e desenvolvimento de uma estação meteorológica remota automatizada. O objetivo é tornar possível e eficiente o fornecimento de informações meteorológicas para o atendimento de comunidades carentes e regiões com altos índices de catástrofes naturais, como chuvas fortes que possam causar possíveis deslizamentos de terras. O sistema permite que sejam 
tomadas ações antecipadas de alerta e evacuação dos locais monitorados. Tal solução é implementada com a utilização da tecnologia RF Link (transmissão por radio frequência), aliada a um microcontrolador, no qual serão construídas duas estações, uma para coletas e outra para transmissões de informações, como: temperatura ambiente, umidade relativa do ar, altitude, pressão atmosférica e recepção/monitoramento das mesmas.

Silva (2017) apresenta um projeto baseado na tecnologia Arduino, criado com sensores de baixo custo, com a facilidade de investir no setor produtivo e a comunidade acadêmica. Onde, foram obtidos e analisados 100 horas de dados para a Estação Automática Arduino e do INMET (referência), no mesmo ponto na cidade de Maceió/AL, durante os dias 06 de setembro a 11 de setembro de 2017. Ao ser validados os dados obtidos pela Estação Automática Arduino, mostra-se sua eficiência, tendo como limitação o fato da necessidade de alimentação através de energia elétrica. Foram realizados medidas de cinco variáveis meteorológicas: Temperatura, Pressão, Umidade, Velocidade do Vento e Precipitação. As análises foram satisfatórias, concluindo-se que a Estação Automática Arduino, pode ser utilizada para fins de pesquisas e análises de microclimas.

Verifica-se que muitos trabalhos criam estações meteorológicas voltadas para o monitoramento das variáveis de temporais, enquanto este trabalho tem como objetivo construir e desenvolver um protótipo de hardware voltado para a automação de sistemas de irrigação por microaspersão a partir do monitoramento de variáveis ambientais para plantações de açaí.

\section{Metodologia}

\subsection{Descrição da área de estudo}

A pesquisa será desenvolvida no município de Cametá, PA. O município está localizado na microrregião de Cametá, mesorregião do nordeste paraense, Estado do Pará, abrangendo uma área de $3.112 \mathrm{~km} 2$, situando-se entre as coordenadas de $01^{\circ} 55^{\prime} 00^{\prime \prime} \mathrm{e}$ 02'38'25" latitude sul e de 49 50'34" e 49 $11^{\circ}$ '13" de longitude oeste de Greenwich, limitando-se a oeste pelo município de Oeiras do Pará, a leste pelo município de IgarapéMirí, ao sul pelo município de Baião e ao norte pelo município de Limoeiro do Ajuru (conforme Figura 1).

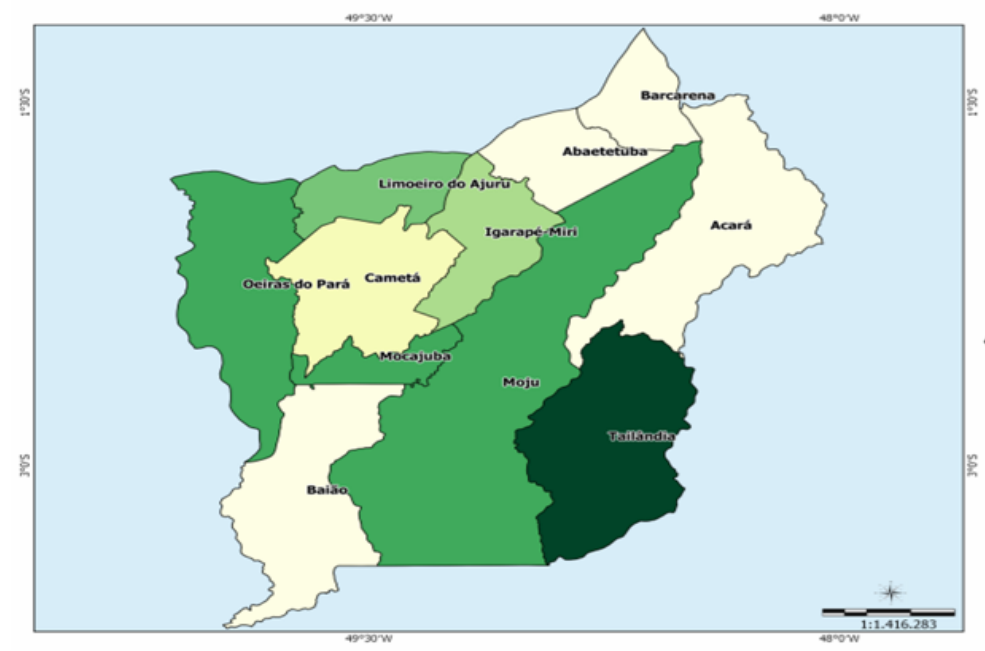

Figura 1 - Mapa de Cametá com os municípios limitantes. Fonte: IBGE. 


\subsection{Sistema de Irrigação}

Para a cultura do açaí, o sistema de irrigação mais adequado é o de microaspersão. Este tipo de emissor possui raio de ação que contempla o espaçamento da cultura e são menos propensos ao entupimento quando comparados aos emissores por gotejamento (HOMMA et al.2009). Este sistema utiliza emissores que distribuem gotículas de água de modo mais suave e uniforme, através de asas giratórias ou "bailarinas", e podem trabalhar apontados para cima ou para baixo.

A escolha deste sistema por microaspersão levou em conta o raio de alcance ("raio molhado") na pressão de trabalho, sugerida pelo fabricante, e um mínimo de $30 \%$ de área molhada do espaço ocupado pela planta.

\subsection{Hardware necessário}

Como critério para escolha dos hardwares que serão utilizados neste projeto, é necessário entender o conceito de Open Hardware, que são circuitos eletrônicos ou hardware de computador que podem ser copiados livremente, já que o próprio desenvolvedor disponibiliza o diagrama esquemático, lista de componentes, layout de placa e outras informações relacionadas ao hardware (THOMSEN, 2014).

Ainda segundo Thomsen (2014), a redução de custos é uma das primeiras vantagens que todo desenvolvedor pensa, entretanto outra grande vantagem é a possibilidade de melhorar o hardware, acrescentando alguma funcionalidade extra ou até mesmo resolvendo algum problema de funcionamento no hardware original, motivo pelo qual este trabalho selecionou o Arduino como parte do projeto proposto.

\subsubsection{Arduino e Sensores}

Arduino é uma placa computacional que torna os computadores capazes de detectar e controlar outros hardwares através de portas analógicas e digitais. É uma plataforma open-source de computação baseada em um microcontrolador inserido em uma placa relativamente simples, além de um ambiente de desenvolvimento específico para o desenvolvimento de softwares embarcados.

O hardware é composto de um processador Atmel AVR, um cristal oscilador e um regulador linear de 5 volts. A placa expõe os pinos de entrada e saída em um encaixe padrão para que se possa conectar circuitos externos que agregam novas funcionalidades ao projeto (MCROBERTS, 2011).

Para este trabalho pretende-se trabalhar com o Arduino Pro Mini (Figura 2), que é um microcontrolador baseado no ATMega328 que roda a $16 \mathrm{Mhz}$ e 5v. Possui 14 saídas/entradas digitais (das quais 6 podem ser usadas como saídas $P W M$ ), 8 entradas analógicas e botão de reset.

\footnotetext{
' Open source é um termo em inglês que significa código aberto. Isso diz respeito ao código-fonte de um software, que pode ser adaptado para diferentes fins.
} 


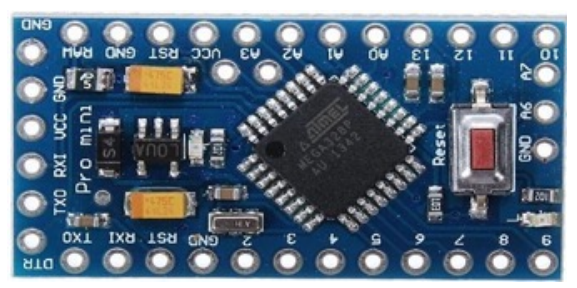

Figura 2 - Placa arduino pro mini.

Fonte: (Arduino, 2018)

O Arduino Pro Mini foi planejado para instalações semi-permanentes e acompanha 3 barramento de pinos que não estão soldados na placa, permitindo assim o uso de vários tipos de conectores de encaixes fáceis, jumpers.

É possível inserir novas funcionalidades à placa Arduino conectando placas adicionais chamadas Shields2. Estas podem ser ligadas sobre a placa de circuito impresso do Arduino com a finalidade de se expandir sua capacidade (ARDUINO, 2018).

As Shields segue a mesma filosofia da ferramenta original: são fáceis de montar e possuem baixo custo de produção (ARDUINO, ESCUDO ETHERNET ARDUINO, 2018). Destacando-se que existem módulos para as mais variadas finalidades, de acordo com a necessidade do projeto.

Para a estação meteorológica proposta será necessário sensores que deverão ter como interface a placa Arduino. Inicialmente prever-se um sensor de umidade e temperatura do ar, que permitirá a realização de leituras de temperaturas entre -40 a +80 graus Celsius e umidade entre 0 a $100 \%$. É prevista também a necessidade de comunicação com outros tipos de hardware mais potentes, como é o caso do Raspberry PI (FILIPEPLOP, 2018).

Um sensor que deverá também ser utilizado neste projeto é o sensor de umidade do solo, higrômetro. Este sensor foi feito para detectar as variações de umidade no solo, indicando quando o solo está seco e quando está úmido (FILIPEFLOP, 2018).

\subsubsection{RaspBerry Pi}

Raspberry $P i$ é um computador de baixo custo e que tem o tamanho de um cartão de crédito, foi desenvolvido no Reino Unido por uma fundação de igual nome. De maneira geral, para usá-lo, basta plugar um teclado e um mouse padrão a ele e conectá-lo a um monitor.

Entende-se que o mini computador citado poderá realizar os processamentos de dados mais pesados da estação, já imaginando-se a possibilidade de expansão da quantidade de sensores utilizados, aumento de processamentos de dados necessários assim a expansão/evolução geral do sistema.

Considerado um modelo versátil, o Raspberry Pi 3 modelo B, é utilizado para várias soluções computacionais (SOUZA, 2018), como:

- Living Room PC: É possível ligá-lo à TV e desfrutar de uma experiência de minimídia com $O p e n E L E C$ e você vai ter um cinema em sua casa. 
- Retro Pi: é possível instalar um sistema específico e transformar o equipamento em um vídeo game com jogos antigos.

- Estudos sobre Programação: é possível instalar uma grande quantidade de ambientes de programação para alunos iniciantes.

O modelo do RaspBerry Pi 3, é o modelo a ser incorporado a proposta desta pesquisa, dado que esta versão mais atual do dispositivo é mais poderosa, contando assim com mais memória de leitura e poder de processamento, que são respectivamente $1 \mathrm{~GB}$ e processador de quatro núcleos de $1.2 \mathrm{GHz}$, Figura 2.

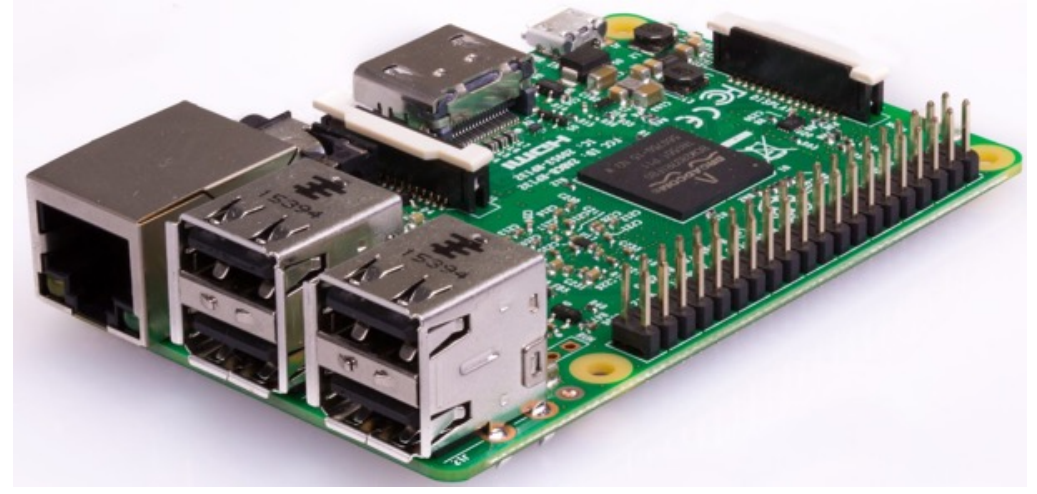

Figura 1 - RaspBerry Pi 3.

Fonte: (Raspberry, 2018).

\subsection{Módulos propostos}

Este projeto é voltado para produtores de pequeno a médio porte, por isso os componentes de software e hardware pensados na composição do sistema proposto buscam oferecer o melhor custo versus benefício possível para este público alvo, ou seja, no projeto prevêse a utilização de tecnologias livres voltadas para hardware e software que permitirão o barateamento da produção do sistema.

Relacionado a hardware, prevê-se a utilização de componentes do projeto Arduino (Stevan e Adamshuk, 2015) juntamente conectados a componentes do projeto Raspberry PI (Monk, 2013), já para softwares prevê-se o desenvolvimento de programas embarcados capazes de controlar todas as partes do sistema e que podem ser disponibilizados a partir de licenças livres, porém deve-se destacar que tais programas serão implementados utilizando-se conhecimentos de Programação Embarcada e até mesmo Inteligência Artificial de computadores, ou seja, são programas de complexidade elevada que permitirão ao sistema a tomada de decisões inteligentes, vindo assim a cumprir com seu objetivo maior.

Para entender como o sistema de controle de irrigação por microaspersão é constituído, primeiramente precisa-se compreender como são constituídos seus principais módulos, que são: Módulo de Controle Central - MCC, Módulo de Monitoramento Ambiental - MMA, Módulo de Armazenamento - MA, Módulo Energético - ME e Módulo de Irrigação - MI.

Cada um dos módulos citados acima possui diferentes finalidades para o sistema, porém se comunicam de maneira integrada a fim de permitir que este venha a cumprir seu objetivo maior. 
Para um melhor entendimento sobre a disposição de cada módulo do sistema, segue uma ilustração deste, Figura 3. Vale ressaltar que seus componentes internos são descritos nas sessões seguintes.

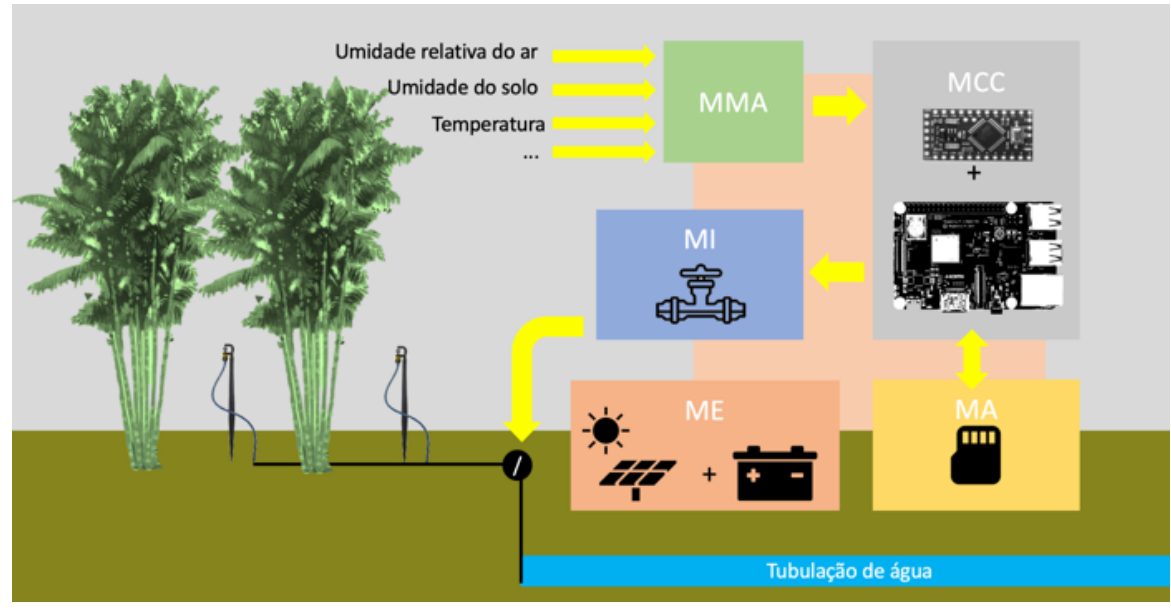

Figura 3 - Ilustração dos módulos utilizados na proposta pretendida.

Fonte: Os Autores.

\subsubsection{Módulo de Controle Central - MCC}

O MCC do sistema é responsável por realizar o controle de abertura e fechamento de válvulas hidráulicas ligadas diretamente ao sistema de irrigação. Para isso, esta unidade recebe dados oriundos de variáveis ambientais provenientes do Módulo de Monitoramento Ambiental - MMA.

A tecnologia de hardware empregada neste módulo, de maneira simplista, se define em uma placa de Arduino mini Pro e uma mini-pc RaspBerry PI 3, que funcionam em conjunto e são capazes de atuar como uma unidade de processamento central para sistema, onde o Raspberry Pi 3 funciona como unidade de processamento e inteligência e o Arduino como unidade auxiliar de processamento e controle.

Raspberry PI 3, principais características: Clock de Processamento: $1.2 \mathrm{GHz}$; GPU: Video Core IV; RAM: 1GB; Wireless: 802.11n; Bluetooth: 4.1; e Armazenamento: $S D$.

Placa Arduino Pro Mini, principais características: Clock de Processador ATMega328 que roda a 16Mhz e 5v. Possui 14 saídas/entradas digitais (das quais 6 podem ser usadas como saídas $P W M), 8$ entradas analógicas e botão de reset.

Observação, o principal objetivo de se ter duas placas controladores centrais é a possibilidade de desligar em alguns momentos o Raspberry Pi para assim consumir menos energia.

\subsubsection{Módulo de Monitoramento Ambiental - MMA}

Esta unidade do sistema é responsável por realizar a captura dos dados oriundos do ambiente através de sensores que acompanham em tempo real as mudanças climáticas do microambiente de dentro da propriedade rural a qual o sistema está inserido. As principais variáveis ambientais monitoradas são: umidade do solo, umidade relativa do ar e temperatura. 
Juntamente com as principais variáveis ambientais listadas acima, este módulo também prevê o monitoramento de variáveis secundárias, com a finalidade servir de base para explorações mais aprofundadas, a nível de mineração de dados.

Variáveis secundárias que serão utilizadas: Pressão atmosféricas; Ultravioleta; Sensor de corrente; Sensor de fluxo de água; Sensor de nível de água; Sensor de luminosidade; Sensor de chuva; e Sensor de $\mathrm{CO}_{2}$.

\subsubsection{Módulo de Armazenamento - MA}

Esta unidade do sistema é responsável por realizar o armazenamento local dos dados coletados pelos sensores. De maneira técnica, trate-se de um módulo que realiza a persistência de dados em uma unidade $S D$ (do inglês, Secure Digital Card), instalada no dispositivo, que pode ser removida do sistema como um todo a fim de ser descarregada para um computador pessoal a fim de se viabilizar a realização de estudos e análises aprofundadas sobre os dados monitorados.

\subsubsection{Módulo Energético - ME}

Mesmo levando em conta que nas propriedades rurais onde pretende-se realizar a instalação de unidades demonstrativas de protótipos desta proposta possuem energia elétrica, deve-se levar em conta que em projetos de automação são necessários alternativas para lidar com problemas corriqueiros como falta de energia. Diante deste cenário, o módulo energético prevê a utilização de painéis solares que permitam a alimentação energética do protótipo.

Principais itens deste módulo: Bateria selada 12 volts e 7 ah; e Painel de energia solar fotovoltaico $12 \mathrm{v}$ e 22 watts.

\subsubsection{Módulo de Irrigação - MI}

O módulo de irrigação é composto por um sistema elétrico baseado em relés que interliga válvulas hidráulicas ao módulo de controle central. Os principais componentes deste módulo são: Válvulas solenoides de 12 volts; e Relés 12 volts de 10 amperes.

\section{Resultados}

Esta pesquisa, até o presente momento, encontra-se com resultados preliminares referentes ao levantamento de viabilidade do projeto proposto. Com isso, considera-se tal proposta como viável, diante ao contexto apresentado, onde a partir de seu melhoramento e prototipação poderá ser encarada como uma realidade para o seu público alvo, ou seja, médios e pequenos agricultores da região norte.

Desenvolver um protótipo capaz de gerenciar o sistema de irrigação por microaspersão de forma automatizada é um desafio considerável. Desta forma, deve-se destacar que esta pesquisa em seu futuro pretende produzir patentes de hardware e software, assim como produzir uma tecnologia eficiente e com qualidade que irá contribuir para um melhor desenvolvimento de cultivos que utilizam sistemas irrigados.

A divisão inicial do sistema como um todo em cinco módulos, possibilita um melhor entendimento do mesmo, assim como uma melhor especialização de cada módulo no processo que cada um deles devem realizar. Assim como possibilitará uma melhor manutenção e rastreabilidade de problemas quando este sistema estiver implementado. 
A partir do momento que se tenha o protótipo desenvolvido, esta pesquisa pretende realizar os testes de funcionamento para obter a leitura das variáveis ambientais citadas, bem como parametrizar e operacionalizar a miniestação para controlar o volume de água adequado ao período especifico do ano.

\section{Conclusão}

O protótipo pretende automatizar com baixo custo beneficio, uma miniestação equipada com sensores de umidade do solo, de umidade relativa do ar e de temperatura do ar para controlar um sistema de irrigação no cultivo de açaí no sentido minimizar perda na produção do mesmo. Sendo totalmente viável a partir do apresentado por esta pesquisa em seus estados iniciais.

Uma miniestação com microcontrolador integrado como gerenciador de irrigação, pode subsidiar pequenos agricultores com baixo investimento além de possibilitar aumento de produção e também pode colaborar na produção continua do açaí.

Os componentes utilizados em cada um dos módulos presentes no sistema como um todo, se mostram capazes de realizar o processamento desejável de maneira promissora, além de ainda permitir a expansão do sistema para novas funcionalidades futuras.

O custo de implantação do projeto é algo que deve ser levado em consideração no processo de desenvolvimento dos protótipos almejados por esta pesquisa. Por isso, a partir dos primeiros protótipos, deverá se analisar meios de baratear o projeto como um todo.

Consumo energético é um fator que deve ser levado em consideração desde o início do projeto, pois como descrito anteriormente o módulo elétrico possui uma bateria e painéis solares, que apesar da considerável autonomia da bateria em questão, em períodos de inverno o seu carregamento solar poderá ser baixo.

\section{Referencias}

ARDUINO. (2018). ARDUINO. Acesso em 2018 de Março de 02, disponível em ARDUINO: http://www.arduino.cc/en/Main/arduinoShields

ARDUINO. (2018). ESCUDO ETHERNET ARDUINO. Acesso em 2018 de Março de 02, disponível em ESCUDO ETHERNET ARDUINO: http://www.arduino.cc/en/Main/ArduinoEthernetShield

Arduino Store. (2018). Arduino Store. Fonte: Arduino Store: https://store.arduino.cc/usa/new-products

COSTA, M. D. (2013). Central meteorológica microcontrolada de baixo custo. Curso Superior de Tecnologia em Automação de Processos, Trabalho de Conclusão de Curso. Pato Branco, Paraná, Brasil: Universidade Tecnológica Federal do Paraná.

FILIPEFLOP. (2018). Sensor de Temperatura. Acesso em 2018 de Junho de 30, disponível em Filipeflop: https://www.filipeflop.com/produto/sensor-deumidade-e-temperatura-am2302-dht22/ 
FILIPEPLOP. (2018). Sensor Umidade. Acesso em 2018 de Junho de 4, disponível em Filipeflop: https://www.filipeflop.com/produto/sensor-de-umidade-do-solohigrometro

HOMMA, A. K. (1993). Extrativismo vegetal na Amazônia: limites e oportunidades. Brasília-DF: Embrapa.

HOMMA, A. K., CARVALHO, J. E., MENEZES, A. J., FARIAS NETO, J. T., \& MATOS, G. B. (2009). Custo operacional de açaizeiro irrigado com microaspersão no Município de Tomé-Açu. Em A. K. HOMMA, J. E. CARVALHO, A. J. MENEZES, J. T. FARIAS NETO, \& G. B. MATOS, Emprapa Amazônia Oriental (p. 5). Belém: Comunicado Técnico.

MCROBERTS, M. (2011). Arduino Básico. Novatec.

MONK, S. (2013). Programando o Raspberry PI. ISBN: 978-85-7522-357-4. Novatec.

RASPBERRY, P. (2016). Raspberry Pi 3. Fonte: Raspberry Pi: http://www.sinect.com.br/2016/selecionados.php

ROSITO, J. P. (2014). Estação inteligente de coleta e transmissão de dados meteorológicos em áreas de risco. Brasilia, Brasil: Centro Universitário de Brasília.

S., G. G., \& LIMA, J. J. (2009). Alteraçoes no espaço urbano de Cametá e os impactos ajusante da UHE Tucurí. Em G. G. S., \& J. J. LIMA, Cidades na Floresta. São Paulo: CASTRO. E.

SILVA, M. C., \& all, a. (2017). Estudo de variáveis meteorológicas para análise de microclima, usando tecnologia de baixo custo. $X$ Workshop Brasileiro de micro meteorologia. Santa Maria.

SOUZA, L. (2018 de Outubro de 2018). 25 Projetos par se fazer com a Raspberry PI 3. Fonte: loja Mundi: https://www.lojamundi.com.br/blog/25-projetos-incriveisraspberry-pi-3.html

STEVAN, L., \& ADAMSHUK, R. (2015). Automação e Instrumentação Industrial com Arduíno. Teoria e Projetos. ISBN-10: 8536514787. Saraiva.

THOMSEN, A. (07 de Outubro de 2014). Open Hardware. Acesso em 30 de Janeiro de 2018, disponível em Filipeflop: https://www.filipeflop.com/blog/open-hardwarelivre/ 